\title{
Magnetic Micro-Barcodes for Molecular Tagging Applications
}

\author{
T.J. Hayward ${ }^{1}$, B. Hong ${ }^{1}$, K. N. Vyas ${ }^{1}$, J.J. Palfreyman ${ }^{1}$, J.F.K. Cooper ${ }^{1}$, Z. Jiang ${ }^{1}$, \\ J.R. Jeong ${ }^{2}$, J. Llandro ${ }^{1}$, T. Mitrelias ${ }^{1}$, J.A.C. Bland ${ }^{1}$ and C.H.W. Barnes ${ }^{1}$ \\ ${ }^{1}$ Cavendish Laboratory, University of Cambridge, Cambridge, UK \\ ${ }^{2}$ Department of Materials Engineering, Chungnam National University, Daejeon, South Korea
}

\begin{abstract}
$\underline{\text { Abstract }}$
We describe proof-of-principle experiments and theory that demonstrate a new method of performing multiplexed biological assays by using microscopic tags which carry multi-bit magnetic codes to label probe biomolecules. It is demonstrated that these "micro-barcode tags" can be encoded, transported using micro-fluidics and are compatible with surface chemistry. We also present simulations and experimental results which suggest the feasibility of decoding the micro-barcode tags using magnetoresistive sensors. Together, these results demonstrate substantial progress towards meeting the critical requirements of a magnetically encoded, high-throughput and portable biological assay platform. We also show that an extension of our technology could be potentially used to label libraries consisting of $\sim 10^{4}$ distinct probe molecules, and could therefore have a strong impact on mainstream medical diagnostics.
\end{abstract}

\section{$\underline{\text { Introduction }}$}

Multiplexed biological assays allow biological samples to be screened for multiple types of target molecules simultaneously and are a key tool in contemporary medical diagnostics, research and drug development. In the conventional, flat-array approach, which is typified by microarray technology, assays are multiplexed by concentrating probe biomolecules into small spots on a planar surface. A signal (generally optical) originating from these spots then allows the presence or absence of target molecules to be determined. This approach to multiplexing is also used in recently proposed "magnetic biochip" technologies [1-4], where target molecules are detected when they mediate binding between magnetic microspheres and high-sensitivity stray field sensors.

More recently, an alternate approach to multiplexing assays, known as Suspension Assay Technology (SAT), has been developed [5]. In SAT binding assays take place at the surfaces of suspended micron-scale tags that carry a characteristic signature to encode the identity of the specific probe biomolecules attached to their surface. By incorporating several subsets of particles with 
distinguishable signatures into an assay it is possible to screen for several targets simultaneously, and hence perform a multiplexed assay. Suspension assays benefit from the high reaction speed associated with solution-based kinetics, high throughput via the use of flow-cytometry techniques, easy modification of probe sets and low assay cost due to the possibility of batch-fabricating tags [5]. Although planar arrays are likely to remain the tool of choice when dealing with extremely large probe sets, SAT is considered to be advantageous in circumstances where a modest numbers of probes are to be detected in large populations or when flexibility in the probe set used is desired.

The tags in any SAT must possess four critical properties: firstly, it must be possible to encode to the tags with information that can be used to identify the attached probe molecule. This can either be done during the fabrication process or, ideally, post-fabrication for maximum flexibility. Secondly, it must be possible to transport the tags using micro-fluidics. This is essential if the assay platform is to be capable of high through-put. Thirdly, it must be possible to attach probe molecules to the tags' surface. Finally, it must be possible to read back the information encoded to the tags in a rapid and reliable fashion.

Although a number of SAT platforms which meet the above criteria have been suggested, almost all encode the identity of the probe molecules optically, using either a spectral [6-8] or graphical [9-12] signature. Reading these signatures generally requires bulky and expensive optical and CCD equipment, and furthermore, automation of the reading requires computationally expensive image processing. This suggests that such technologies are not amenable to the kind of miniaturisation that is required to create a platform which can be used outside of a laboratory environment. Furthermore, even in the case of the more simple spectral encoding technologies [6], which are more easily miniaturised than graphical encoding technologies, the number of available codes is limited to $<100$ by spectral overlap.

A solution to these limitations would be to create tags carrying information that could be read back directly into an electrical signal. Optical techniques would then only be required to detect binding between probe and target molecules (most likely using conventional fluorescent labels). Because this is possible using a simple optical detector such as a photodiode [13], the bulky imaging optics and image analysis systems required by the technologies described above would not be necessary. Furthermore, the separation of the signals used to identify binding from those used to identify the probe molecules would remove interfere between the two and allow large probe-sets to be analysed simultaneously. Such a technology would therefore allow the creation of a highly-parallel miniaturised SAT platform ideal for performing multiplexed screening in the field or at the point-ofcare.

Here, we propose a new form of SAT in which each tag carries a microscopic magnetic barcode that encodes the identity of an attached biomolecule. Potentially, the magnetic fields created by these 
micro-barcodes can be detected using microscopic magnetoresistive sensors, and hence the tags meet the detection criteria described above.

A schematic diagram of the magnetic tags we propose is shown in Figure 1. The tags consist of a number of rectangular thin-film ferromagnetic elements that are supported by a microscopic SU8 polymer substrate. Because the ferromagnetic elements are elongated, magnetic shape anisotropy stabilises magnetisation states where the magnetisation lies dominantly along their long axis. Each element can therefore support one of two opposing magnetisation states (i.e. up magnetisation or down magnetisation), and these two configurations are assigned binary 0 and binary 1 respectively, so that the collective states of the magnetic elements form a binary code written along the SU8 support. The $N$ bits of the tag offer a total of $2^{N}$ distinct codes which can be used to encode the identities of attached probe biomolecules. The polymer substrate encloses the magnetic elements, both protecting the magnetic elements from damage by the solution in which the tags are immersed and lowering the tags density such that they will float.

In this paper we demonstrate that the micro-barcode tags can be encoded with information, transported using micro-fluidics and are compatible with surface chemistry. We also present simulations and proof-of-concept experimental data which suggest the feasibility of decoding the micro-barcode tags using conventional magnetoresistive sensors. Cumulatively, our results indicate that magnetic micro-barcode tags have great potential for use in a high-throughput portable SAT platform.

\section{$\underline{\text { Experimental }}$}

An optical microscope image of a magnetic micro-barcode tag is shown Figure 2(a). Each tag consists of a series of five $20 \mathrm{~nm}$ thick $\mathrm{Ni}_{80} \mathrm{Fe}_{20}$ rectangular magnetic elements, encapsulated within an SU8 support, which has a length of $100 \mu \mathrm{m}$, a width of $30 \mu \mathrm{m}$ and a thickness of $2 \mu \mathrm{m}$. The magnetic elements, which are spaced $20 \mu \mathrm{m}$ apart, are $20 \mu \mathrm{m}$ long, and have widths of 6, 2.5, 2, 1.5 and $1 \mu \mathrm{m}$. These widths were chosen so as to achieve maximum separation between the individual bits' switching distributions as is described in detail in [14].

The micro-barcode tags were fabricated by a five step process. First a $\sim 250 \mathrm{~nm}$ thick Al release layer was thermally evaporated onto a 3 inch diameter silicon wafer. The wafer was then spin-coated with a $1 \mu \mathrm{m}$ thick layer of SU8 resist, which was exposed and hard-baked to cross-link and form the bottom layer of the tags' support.

To define the magnetic elements, polymethylmethacrylate (PMMA) resist was spin-coated onto the hardened SU8 layer and patterned using deep ultra-violet optical lithography, creating a resist template of the elements. A $20 \mathrm{~nm}$ thick $\mathrm{Ni}_{80} \mathrm{Fe}_{20}$ film was then thermally evaporated onto the template and the fabrication of the magnetic elements was completed using lift-off processing. 
To encapsulate the magnetic elements a further $2 \mu \mathrm{m}$ thick SU8 layer was spun onto the substrate and patterned to the desired dimensions of the tags' support using optical lithography. The patterned SU8 surface was then ion milled to a depth of $1 \mu \mathrm{m}$, resulting in an array of completed tags attached to the $\mathrm{Al}$ release layer. Finally, the release layer was dissolved in MF319 developer, leaving the microbarcode tags free-floating in suspension (Figure 2(b)). Further details of this fabrication process, which allows $\sim 10^{6}$ individual tags to be produced simultaneously, can be found in [14].

Magnetic imaging of the magnetic barcode tags was achieved using a commercial magneto-optic Kerr effect microscope produced by NEOARK. An electromagnet allowed in-plane magnetic fields of up to $1 \mathrm{kOe}$ to be applied with the sample in-situ. The images shown in this paper were taken prior to the ion-milling and release stages of the tags processing in order to provide the reader with clear images. However, extensive measurements on released tags showed that these final fabrication steps had no effect on the tags' magnetic properties.

To examine the compatibility of the tags with micro-fluidics a polydimethylsiloxane (PDMS) channel was molded from a SU8 template, which was patterned on a silicon substrate using optical lithography. The channel had a depth of $20 \mu \mathrm{m}$, a width of $100 \mu \mathrm{m}$ and included a short $50 \mu \mathrm{m}$ wide constriction to control the orientation of the tags. To enclose the channel the molded PDMS piece was bonded channel-side-down onto a glass microscope slide. Plastic tubing was used to connect the channel to a vial containing a dilute aqueous suspension of the micro-barcode tags. The vials were then pressurised using a pneumatic pumping system, causing the solution containing the tags to flow through the channel.

As an initial demonstration of the feasibility of coupling molecules to the tags we attached the fluorophore aminomethylfluorescein-hydrochloride (AMF) directly onto the SU8 tags surface. A simple fluorophore was used here as it allowed us to directly assess whether attachment was successful or not via fluorescence microscopy. Wafer pieces were either diced to fit inside $1.5 \mathrm{ml}$ eppendorfs or pre-released tags were used. The tags were submerged in $1 \mathrm{ml}$ dimethylformamide with $1 \% \mathrm{AMF}$ (and $5 \mu \mathrm{l}$ piperidene to neutralise the $\mathrm{HCl}$ ) and mixed for 2 hours at room temperature. Repeated washing was carried out with DMF followed by de-ionised water until the supernatant appeared colourless.

Simulations of the interaction of the micro-barcode tags with a tunnel magneto-resistive (TMR) sensor were performed by using a simple Stoner-Wohlfarth model to describe the sensor's free layer. The behaviour of this model was calibrated to reproduce the behaviour of a sensor which has previously been demonstrated by Shen et al. to be sufficiently sensitive to detect single superparamagnetic beads in micro-fludic flow [15]. Finite element simulations were used to calculate the magnetic field patterns created by the tag's bits, which were assumed to be uniformly magnetised. These field patterns were then applied to the sensor model in such a manner as to represent the 
complete micro-barcode passing the sensor at finite fly-height. Further details of the model used in these calculations can be found in [16].

Attempts were also made to obtain proof-of-concept detection data using a commercial TMR sensor from Micromagnetics Inc [17]. The sensor chip was embedded in a PDMS base and wire bonded to facilitate connection to an external balanced AC bridge. A PDMS channel similar to that shown in Figure 4(a) was then bonded over the device so that the $6 \mu \mathrm{m} \times 2 \mu \mathrm{m}$ sensing area lay in the centre of the $50 \mu \mathrm{m}$ wide constriction. The voltage across the bridge was monitored using a lock-in amplifier attached to a digital oscilloscope. Micro-barcode tags were then flowed over the sensor using the micro-fluidic system described previously.

\section{Results and Discussion}

\section{Encoding the Micro-barcode Tags}

For the micro-barcodes to be useful as a SAT platform it must be possible to encode information to large numbers of them in a rapid and reliable fashion. In conventional data storage media, such as hard disks, each bit is individually and successively switched using a localised magnetic field. However, such an approach is unlikely to be feasible when trying to write large numbers of solutionphase tags, due to the number of degrees of freedom involved.

As a solution to this problem, we have developed a method of encoding micro-barcode tags using magnetic fields that are uniform across the entire tag. Because each of the tag's bits has a different aspect ratio, magnetic shape anisotropy causes each bit to exhibit a different characteristic switching field, with the narrower bits requiring higher fields for a reversal to occur due to their greater anisotropy. Thus, a particular magnetic field strength will align the magnetisations of all elements that have widths above a certain critical value. This is illustrated in Figures 2(c)-(h), which show magneto-optic Kerr effect (MOKE) microscopy images of a micro-barcode tag being written progressively from a (11111) code to a (00000) code using a uniform magnetic field.

A series of $N$ magnetic elements with unique switching fields can be written into any desired configuration by applying a series of $N$ uniform field pulses with varying amplitude and direction [18]. The manner in which this occurs is best explained by example. Here we will consider writing a bit configuration of (01010) into a tag which begins in the (11111) configuration (Figure 3(a)).

Initially a strong negative magnetic field is applied along the long axes of the tag elements. This field is greater than switching field of all of the magnetic elements, and hence the tag is written into the (00000) configuration (Figure 3(b)). Next, a slightly weaker positive magnetic field is applied which is only strong enough to switch the four widest magnetic elements back into a "1" state. The narrowest element remains unperturbed and hence the tag is written into the (01111) configuration 
(Figure 3(c)). A negative magnetic field, again with a smaller magnitude, is then applied. This writes only the three widest bits leaving the tag in the (01000) configuration (Figure 3(d)). The process of applying field pulses with alternating direction and decreasing magnitude is continued until the tag reaches the desired (01010) configuration (Figure 3(e)-Figure 3(f)). Modification of this field sequence allows the tags to be written into any desired configuration. Further details of the magnetic properties of the barcode elements can be found in [14].

The above technique of encoding the tags could be applied prior to their release from the substrate using macroscopic fields or when the tags are in-flow using micro-patterned strip lines (providing that the alignment of the tags can be well regulated). Furthermore, the magnetic switching of micron-scale magnetic elements generally takes place on nanosecond timescales and hence the writing of the tags would have a negligible effect on the throughput of an assay platform.

\section{Microfluidic Transport}

In a portable SAT platform it will be necessary to use micro-fluidic technology to move tags from their point of introduction and through various chambers in which chemical reaction and detection steps will take place. In Figure 4 we demonstrate that such micro-fluidic transport is possible for the micro-barcode tags.

Figures 4(a)-4(c) show optical microscopy images of a magnetic micro-barcode tag flowing through a constriction in a PDMS channel. It can be seen that the tag floats such that it lies parallel to the floor of the channel. Although in the wide sections of the channel the tags tended to cartwheel and flow with random orientation, when flowing through the constrictions their long axes were found to align parallel to their direction of motion as was intended. Obtaining this kind of control of the tags' orientation will be essential when trying to read and write the tags while they are in-flow.

A drawback to using this kind of hydrodynamic focusing to regulate the tags flow is that narrowing the channel with a constriction increases the likelihood of tags becoming stuck and blocking the flow. We observed this several times during our measurements. One possible solution to this problem would be to use a channel that is significantly wider than that which we use here and integrate metallic electrodes into its walls. This would allow us to focus the tags laterally using dielectrophoresis [19], reducing the chances of the channel becoming blocked.

\section{Chemical Compatibility}

The SAT technology we propose in this paper is essentially a generic technology, and for every screening application that it is applied to, a different set of target specific probe biomolecules will need to be attached to the surfaces of the tags. Here, we have coupled the tags to a simple fluorescent dye as an initial demonstration of their chemical compatibility 
The tags' SU8 support provides free surface epoxide groups, onto which covalent bonding chemistry can proceed, usually via a ring-opening reaction with a nucleophilic group such as a primary amine [20]. Therefore, we chose to couple the fluorophore aminomethylfluorescein-hydrochloride (AMF) directly onto the SU8 tags.

Figure 4(d) shows a fluorescence microscope image of free-floating SU8 backbones after attaching the AMF. All of the backbones can be clearly seen to be fluorescent, demonstrating that the SU8 polymer coating offers a route to further chemical modification. The fluorescent intensity of the labelled tags was $\sim 10^{2}$ times greater than that of the unlabelled backbones.

The optimisation of the surface chemistry required to attach specific biomolecular probes, such as single stranded DNA or disease antigens, to the tags will require further work. However, it should also be noted that the tag's SU8 backbone is not the only possible route to chemical functionalisation. During the fabrication of the tags it would be relatively easy to coat one side of the micro-barcode tags with a gold film that would offer excellent chemical compatibility, as we have previously demonstrated [18].

\section{Reading Micro-barcode Tags}

For a SAT platform to achieve high-throughput it is essential that tags can be read while in microfluidic flow. For the micro-barcode tags this necessitates the ability to sequentially measure the states of the tag's magnetic bits using a highly sensitive magnetic field sensor. In the following we use a simple model to demonstrate the feasibility of this.

Shen et al. have previously demonstrated that micron-scale tunneling magnetoresistance (TMR) sensors are capable of detecting individual superparamagnetic microspheres with excellent signal-tonoise ratios [15] and so, as explained previously, we calibrated our model to accurately reproduce the behaviour of this sensor. This is illustrated in Figure 5(a) which compares a transfer curve computed using our model with experimental data from [15].

Initially, single $\mathrm{Ni}_{80} \mathrm{Fe}_{20}$ magnetic bits were simulated passing over the sensor at a range of fly-heights in order to evaluate the detection limits of the system (Figure 5(b)). The sensor was oriented such that it was sensitive to the magnetic field component parallel to the magnetisation of the bits. Signal magnitudes in $\mathrm{mV}$ were estimated by assuming that the sensor was placed in a balanced $\mathrm{AC}$ bridge. The magnetic bits had widths of 1, 2, 3, 4 and $5 \mu \mathrm{m}$, and other dimensions as in the fabricated tags described earlier.

With a fly height of $20 \mu \mathrm{m}$, the signal from the $1 \mu \mathrm{m}$ wide element is $5 \mu \mathrm{V}$, equal to the noise floor of the device demonstrated by Shen et. al. in [15]. However, with fly-heights $<10 \mu \mathrm{m}$ all of the elements are detected with signal-to-noise ratios greater than 4:1, suggesting that micro-barcode tags can be 
read comfortably using contemporary TMR sensors, provided that their fly-height can be kept below this value. Control of the tags fly-height could be exerted using hydrodynamic, magnetophoretic or dielectrophoretic focusing techniques.

Although the simulations described above suggest that the magnetic fields produced by the tags are of sufficient magnitude to permit for the tags to be read successfully it must also be possible to differentiate the sensor responses from each of the tag's bits. Figure 5(c) shows the results of a simulation in which a five-bit micro-barcode was passed across the TMR sensor at a fly-height of 5 $\mu \mathrm{m}$. The simulated tag's elements had widths identical to those in the experimentally fabricated tags. Data for tags carrying the (10101) and (11111) codes is shown. In both cases the MR peaks from each of the five bits are clearly resolved. Further simulations performed using the same tag geometry indicate that all five bits can still be resolved even with fly heights $>10 \mu \mathrm{m}$.

To further demonstrate the feasibility of decoding the tags using magnetoresistive sensors we have also performed proof-of-concept detection measurements using a commercial TMR sensor. Figures 6(a)-6(c) show a sequence of optical microscopy images taken as a tag in the (00000) configuration was flowed over the sensor. The tag can be seen to be passing directly over the sensor, which lies at the apex of the triangle formed by the four gold electronic contacts.

Figure 6(d) shows the signal measured from the AC bridge, along with the predicted response from the model. It appears that there is good agreement between the shapes of the experimental data and that produced by the model. The signal from the $5 \mu \mathrm{m}$ wide (largest) magnetic bit is resolved with a peak-to-peak signal-to-noise ratio of approximately 3:1. However, the noise level is too high for the narrower magnetic bits to be resolved independently.

Despite the high noise levels in our proof-of-concept system we believe that further optimisation will allow signals from all of the bits to be clearly resolved. Our model of the sensor described by Shen et. $a l$. indicates that for a fly height of $10 \mu \mathrm{m}$ the $5 \mu \mathrm{m}$ wide bit should produce a signal of $110 \mu \mathrm{V}$. Combining this with the $5 \mu \mathrm{V}$ (root-mean-square) noise floor quoted by Shen et. al. indicates that their detection system would detect the $5 \mu \mathrm{m}$ wide bit with a signal-to-noise ratio of $\sim 22: 1$, almost an order of magnitude higher than that measured in our proof-of-concept system. An improvement of this nature would allow the sensor responses from the smaller magnetic bits to be resolved easily.

\section{Code Carrying Capacity}

The tags we have discussed thus far are composed of five magnetic bits. These tags are capable of carrying a maxiumum of 32 distinct codes; however for many assays a greater number of biomolecular probes are required. In the following we will use the model described in the previous section to estimate the ultimate code-carrying capacity of the magnetic micro-barcode tags. 
Assuming that the signal from each of the bits is large enough to be resolved above the noise floor of the sensor there are likely to be two factors which affect the number of bits that can be carried by the tags. The first is simply the length of the SU8 backbone. The second is the sharpness of the field profiles produced by the magnetic bits. This affects how closely densely spaced the magnetic bits can be without their sensor responses merging into each other.

The simulations described in the previous section showed that the MR signals produced by the magnetic bits were well described by Lorentzian distributions, the widths of which increased with fly height, but showed only weak dependence on the width of the elements. This indicates that controlling the fly-height of the tags is likely to be the dominant factor in determining how closely the magnetic elements can be spaced. This can be easily understood by considering the limiting cases: In the farfield the magnetic field from a multibit magnetic tag will closely resemble that from a single dipole, while close to the tag the observed magnetic field will depend strongly on the orientation of the magnetisation in the nearest magnetic bit. These effects are described in more detail in [16].

Figure 5(d) shows the calculated code carrying capacity of the magnetic micro-barcode as a function of backbone length and fly-height. These calculations assume that the elements are separated by the full-width-at-half-maximum of the MR peaks produced by the elements at a given fly height. This criterion just allows the peaks from each bit to be independently resolved. A $200 \mu \mathrm{m}$ long tag, a similar size to carriers in other high-throughput SAT platforms [12], can accommodate 13 magnetic bits providing that the fly-height of the tag can be regulated to be $<5 \mu \mathrm{m}$. Such a tag hypothetically offers 8192 unique codes, a similar order of magnitude to that which has been obtained using optical encoding techniques [21]. However, it should be noted that in reality this number of codes can only be achieved if an additional marker is added to define a reference direction for " 1 " and " 0 ". If this is not the case codes such as (10000) and (01111) become degenerate and hence the code carrying capacity is halved for a given tag length.

\section{$\underline{\text { Conclusion }}$}

In this paper we have described a new form of suspension assay technology in which each tag carries a microscopic magnetic barcode that encodes the identity of an attached biomolecule. We have performed initial experiments which demonstrate that the tags can be encoded and decoded, may be transported using micro-fluidics, and are compatible with surface chemistry, thus meeting four critical requirements of a high-throughput SAT platform. We have also estimated the ultimate code-carrying capacity of the tags and have shown that $\sim 200 \mu \mathrm{m}$ long tags could in principle be used to label libraries consisting of over 8000 probe molecules.

In contrast to previously proposed SAT platforms our technology uses micro-patterned magnetic sensors to decode the tags. Such sensors are small, simple and cheap to produce and hence are ideally 
suited to creating portable SAT platforms that could be used in the field or at the point-of-care. Furthermore, unlike simple spectral SATs which may be amenable to miniaturisation [6] than their more complex graphical counterparts, our approach uses different types of signal to detect probetarget binding and to identify probe molecules. This removes interference between the two signals, potentially allowing for large probe sets to be used. We believe that this combination of portability, high-throughput and massive parallelism could have a strong impact on point-of-care medical diagnostics, particularly in cases where a single symptom has large numbers of possible causes.

Further advantages are offered by the writable nature of the micro-barcode tags. With existing SAT technologies creating a library of $N$ codes involves fabricating $N$ batches of tags, as each distinct code requires a slightly different fabrication process. By contrast all micro-barcode tags are nominally identical, and are merely written into distinct configurations. This is likely to offer advantages when dealing with large libraries consisting of hundreds or thousands of probe molecules. Sequentially encoding the tags as the molecules at their surface undergo multiple chemical modifications could also offer a solution to labelling large molecular libraries created by split-and-mix combinatorial chemical synthesis [22].

Despite the successes demonstrated in this paper there are still several challenges that must be overcome. Our detection system must be improved so that the micro-barcode tags can be decoded reliably. One possible way of achieving this would be to locally reduce the fly-height of the tags as they pass the sensor. This could be achieved by integrating metallic electrodes into the roof of the PDMS channels such that the tags are locally pushed down by dielectrophoresis [22]. Further improvements are likely to be possible by improving the performance of our detection electronics, or by employing more advanced signal processing techniques. It will also be necessary to demonstrate the attachment of real biological probes, such as single stranded DNA or disease antigens, to the tags, rather than the simple fluorescent dye used here. More distant goals will involve bringing together the various components of the technology in order to perform proof-of-concept assays with small probe libraries.

The authors acknowledge the Engineering and Physical Science Research Council for financial support. We thank G. Xiao, G.S. Galitonov and D.A. Allwood for useful discussions. This paper is dedicated to the memory of Professor J.A.C. Bland (1958-2007).

[1] D.R. Baselt, G.U. Lee, M. Natesan, S.W. Metzger, P.E. Sheehan and R.J. Colton, Biosensors Bioelectr. 13, 739 (1998)[2] M. Megans and M.W.J. Prins, J. Magn. Magn. Mat. 293, 702 (2005) [3] M.M. Miller, P.E. Sheehan, R.L. Edelstein, C.R. Tamanaha, L. Zhong, S. Bounak, L.J. Whitman and R.J. Colton, J. Magn. Magn. Mat. 225, 138 (2001)

[4] H.A. Ferreira, D.L. Graham, N. Feliciano, L.A. Clarke, M.D. Amaral and P.P. Freitas, IEEE Trans. Magn. 41, 10, 4140 (2005) 
[5] J.P. Nolan and L.A. Sklar, Trends. Biotechnol. 20, 1 , 9-12 (2002)

[6] R.J. Fulton, R.L. McDade, P.L. Smith, L.J. Kienker and J.R. Kettman and J.R. Kettman, Clin. Chem. 43, 9, 1749 (1997)

[7] M. Han, X. Gao, J.Z. Su and S. Nie, Nature Biotechnol. 19, 631 (2001)

[8] F. Cunin, T.A. Schmedake, J.R. Link, Y.Y. Li, J. Koh, S.N. Bhatia and M.J. Sailor, Nature Mat. 1, 39 (2002)

[9] S.R. Nicewarner-Peña, R.G. Freeman, B.D. Reiss, L. He, D.J. Peña, I.D. Walton, R. Cromer, C.D. Keating and M.J. Natan, Science, 294, 137 (2001)

[10] K. Braeckmans, S.C. De Smedt, C. Roelant, M. Leblans, R. Pauwels and J. Demeester, Nature Mat. 2, 169 (2003)

[11] G.S. Galitonov, S.W. Birtwell and N.I. Zheludev, Optics Express 14, 4, 1382 (2006)

[12] D.C. Pregibon, M. Toner and P.S. Doyle, Science 315, 1393 (2007)

[13] K-S. Shin, Y-H. Kim, K-K. Paek, J-H. Park, E-G. Yang, T-S. Kim, J-Y. Kang, and B-K. Ju, IEEE Electr Device L, 27, 9, 746 (2006)

[14] B. Hong, T.J. Hayward, J.-R. Jeong, J.F.K. Cooper, J.J. Palfreyman, T. Mitrelia, A. Ionescu, J.A.C. Bland and C.H.W. Barnes, J. Appl. Phys. 105, 034701 (2009).

[15] W. Shen, X. Liu, D. Mazumdar and G. Xiao, Appl. Phys. Lett. 86, 253901 (2005)

[16] T.J. Hayward, J. Llandro, K.P. Kopper, T. Trypiniotis, T. Mitrelias, J.A.C. Bland and C.H.W. Barnes, AIP Conference Proceedings, 1025, 111-124 (2008)

[17] www.micromagnetics.com

[18] J.-R. Jeong, J. Llandro, Bingyan Hong, T. J. Hayward, T. Mitrelias, K. P. Kopper, T. Trypiniotis, S. J. Steinmuller, G. K. Simpson and J. A. C. Bland, Lab Chip 8, 1883 - 1887 (2008) .

[19] L. Wang, L.A. Flanagan, N.L. Jeon, E. Monuki and A.P. Lee, Lab on a Chip 7, 1114 (2007)

[20] G. Cavalli, S. Banu, R. T. Ranasinghe, G. R. Broder, H. F. P. Martins, C. Neylon, H. Morgan, M. Bradley, and P. L. Roach.. J. Comb. Chem., 9, 3, 462-472 (2007).

[21] P.S. Eastman, W. Ruan, M. Doctolero, R. Nuttall, G. de Feo, J.S. Park, J.S.F. Chu, P. Cooke, J.W. Gray, S. Li and F.F. Chen, Nano Letters, 6, 1059 (2006)

[22] B.J. Battersby, D. Bryant, W. Meutermans, D. Matthews, M.L. Smythe and M. Trau, J. Am. Chem. Soc. 122, 2138 (2000) 


\section{Magnetic Micro-Barcode Tag}

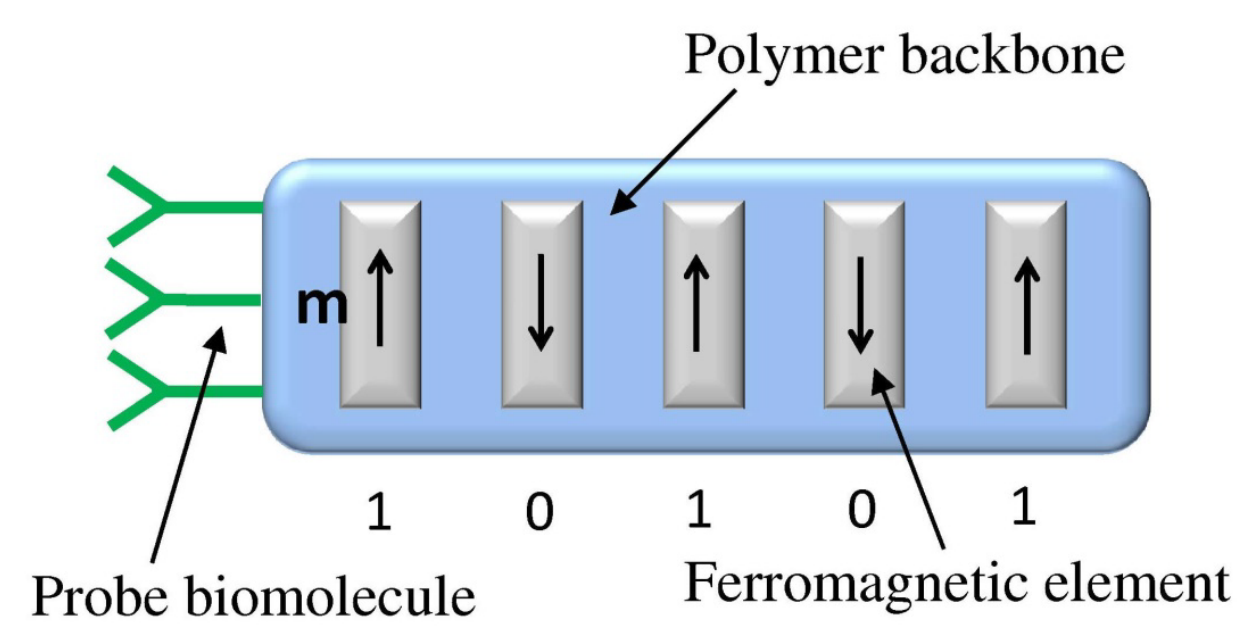

Figure 1: Schematic diagram of a magnetic micro-barcode tag. The tag consists of a series of five ferromagnetic elements which are supported by a polymer backbone. The magnetic elements can support two magnetisation states: up magnetisation (1) or down magnetisation (0). The five bit binary code created by the states of the magnetic elements (i.e. 10101) is used to identify the probe biomolecules attached to the surface of the tag. 
(a)
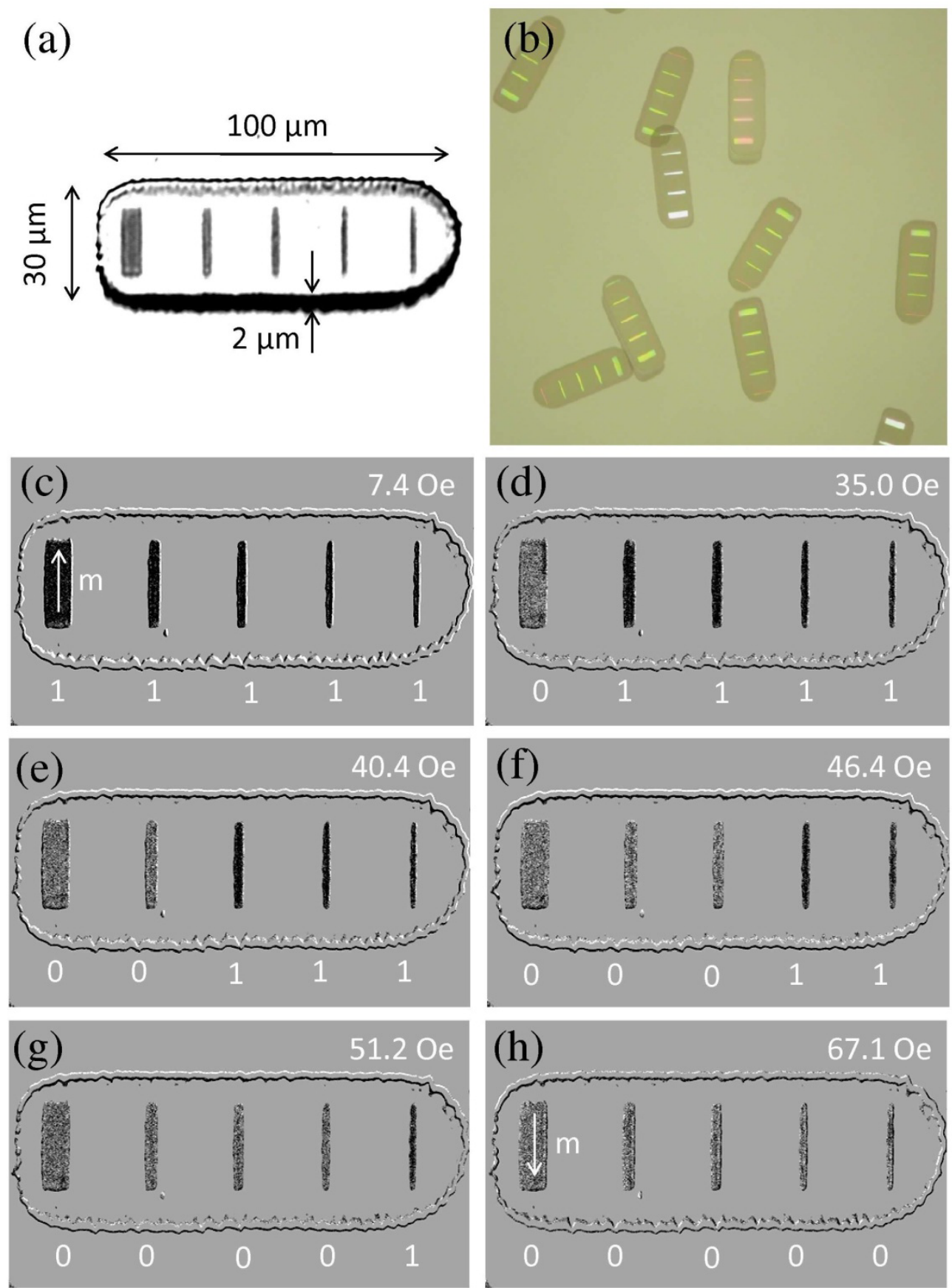

Figure 2: (a) Optical microscope image of a single micro-barcode tag. (b) Optical microscope image of a number of tags in suspension. (c)-(h) Magneto-optic Kerr Effect microscopy images showing a micro-barcode tag being written sequentially from a (11111) code to a (00000) code. Dark contrast corresponds to magnetisation pointing up the page, whereas lighter contrast corresponds to magnetisation pointing down the page. 

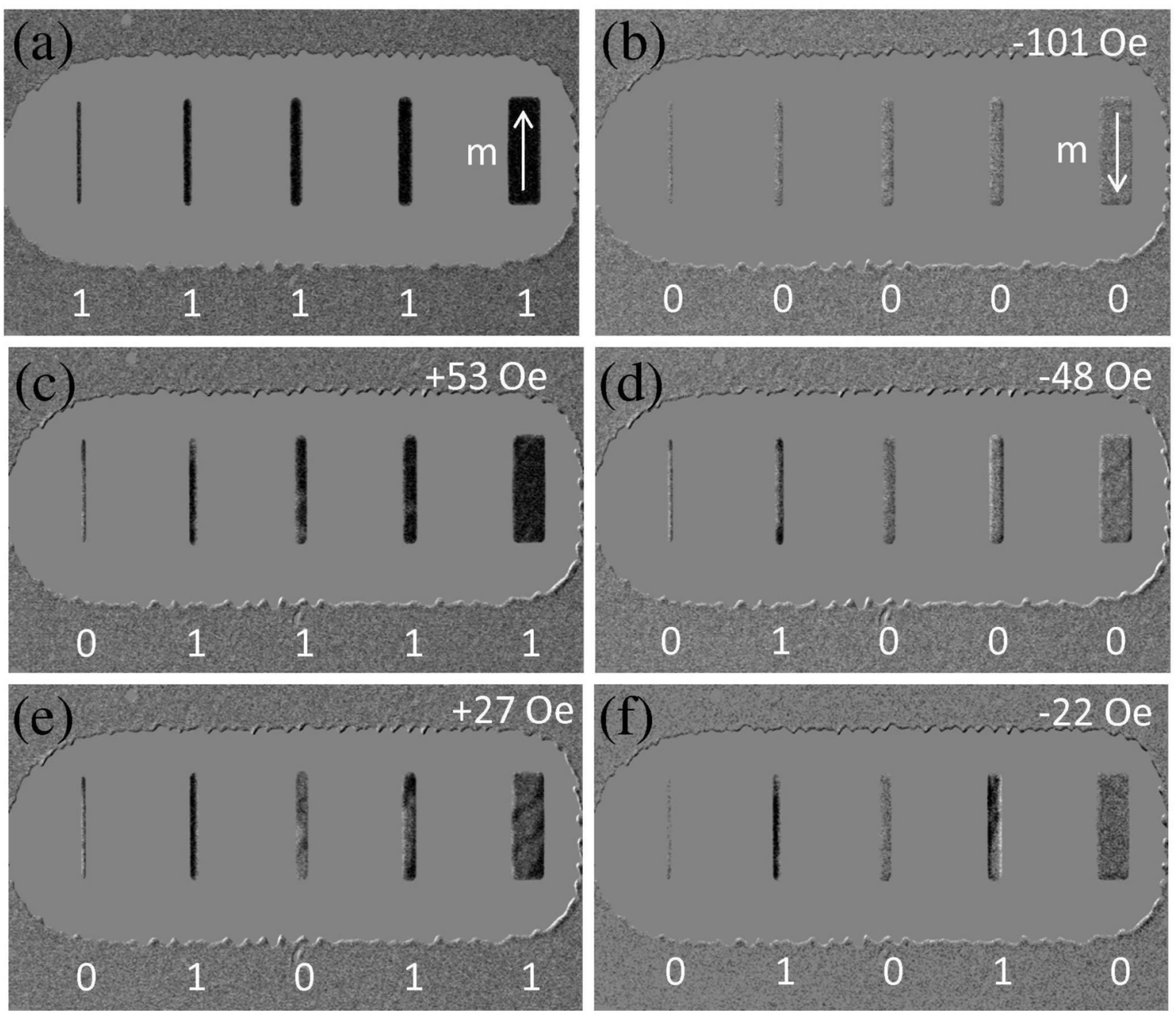

Figure 3: Magneto-optic Kerr Effect microscopy images showing a micro-barcode tag being written from a (11111) code to a (01010) code. Dark contrast corresponds to magnetisation pointing up the page, whereas lighter contrast corresponds to magnetisation pointing down the page. 



Figure 4: (a)-(c) Optical microscopy images showing a micro-barcode tag flowing through a constriction in a $100 \mu \mathrm{m}$ wide micro-fluidic channel. Fluorescence microscopy images of a large number of tags after the attachment of AMF. 
(a)

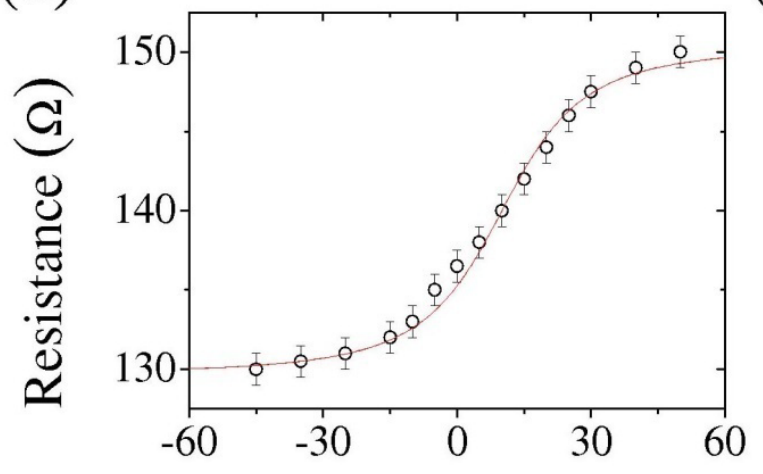

Applied Field (Oe)

(c)

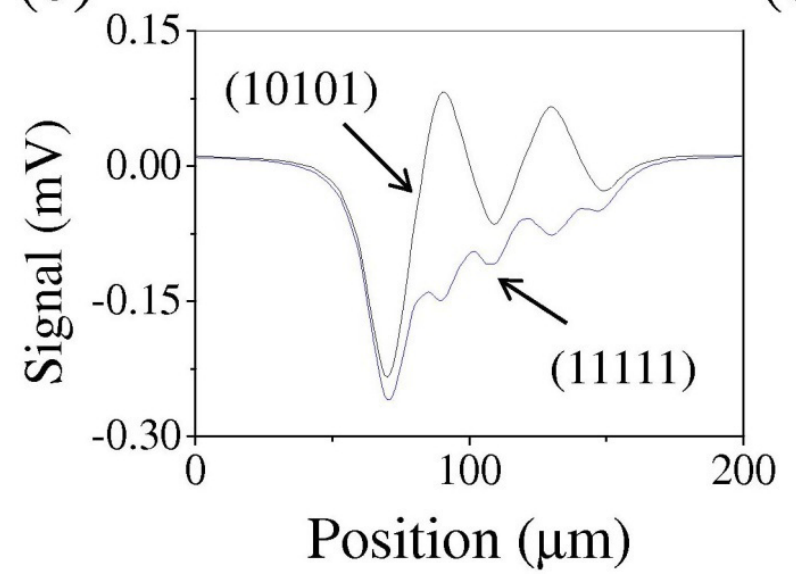

(b)

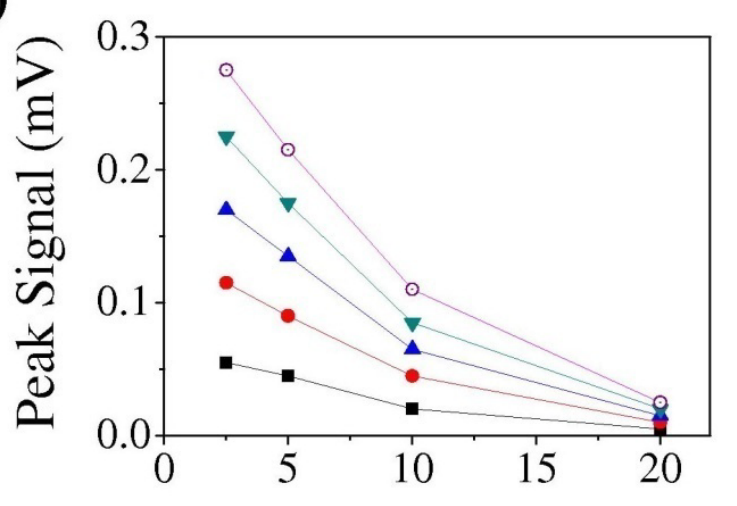

Fly Height $(\mu \mathrm{m})$

(d)

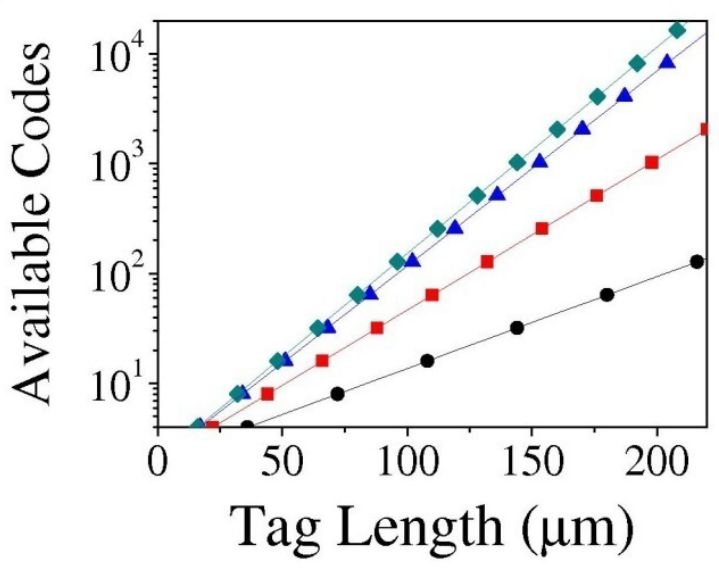

Figure 5: (a) Simulated transfer curve of a Tunnel Magnetoresistance sensor. The open circles show experimental data from [15]. (b) Peak magnetoresistance signals as a function of fly-height produced by $20 \mu \mathrm{m}$ long $20 \mathrm{~nm}$ thick NiFe elements with widths of $5 \mu \mathrm{m}$ (open circles), $4 \mu \mathrm{m}$ (down triangles), $3 \mu \mathrm{m}$ (up triangles), $2 \mu \mathrm{m}$ (closed circles) and $1 \mu \mathrm{m}$ (closed squares). (c) Simulated magnetoresistance signals produced by five-bit micro-barcode tags carrying the (11111) and (10101) codes. The flyheight was $5 \mu \mathrm{m}$. The simulated tag's elements have widths identical to those in the fabricated tags. (d) Number of available codes as a function of tag length for fly-heights of $20 \mu \mathrm{m}$ (closed circles), 10 $\mu \mathrm{m}$ (closed squares), $5 \mu \mathrm{m}$ (up triangles) and $2.5 \mu \mathrm{m}$ (closed diamonds). 

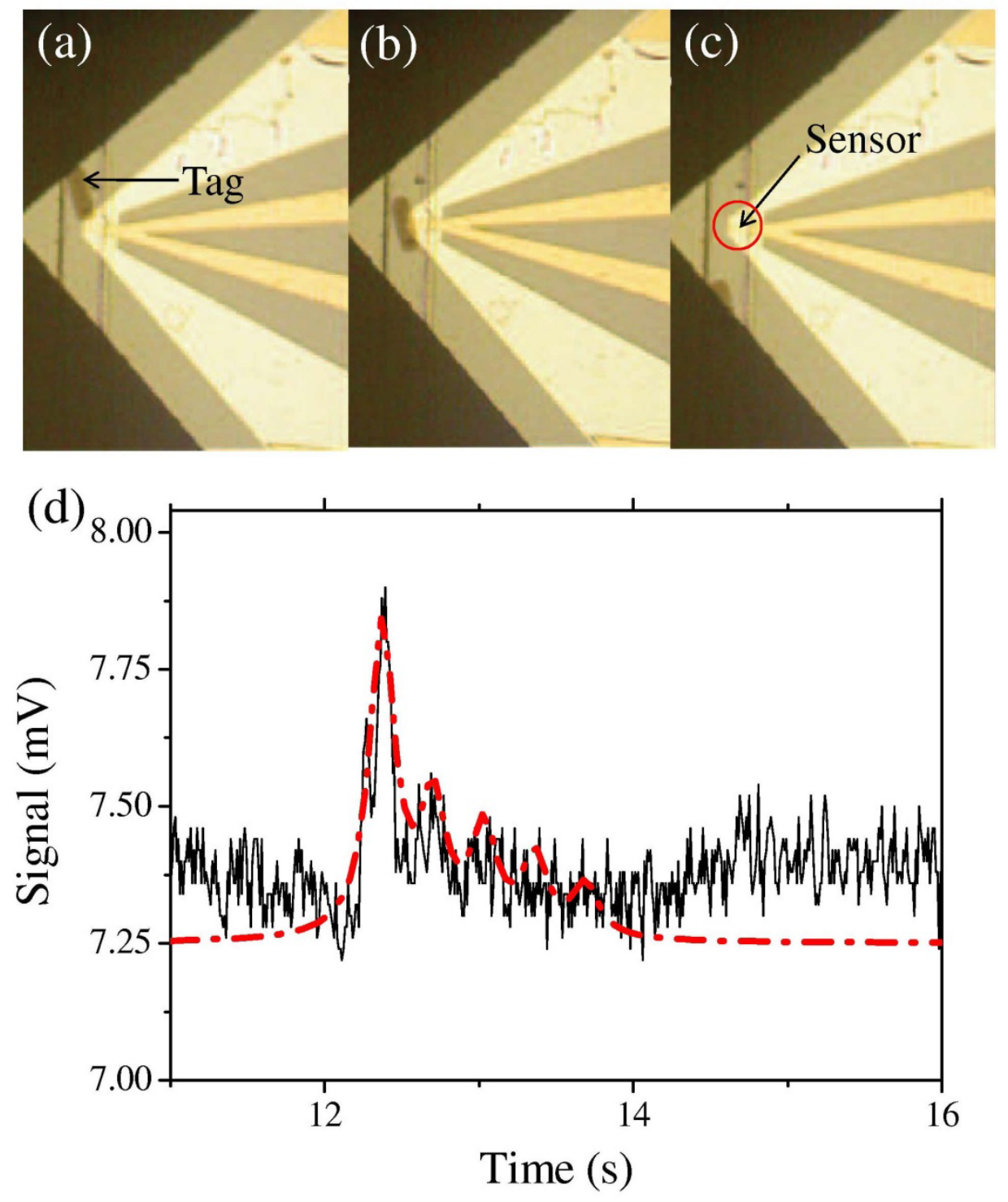

Figure 6: (a)-(c) Optical microscopy images showing the passage of magnetic microbarcode tag over a commercial TMR sensor. (d) Solid line: measured AC bridge voltage as a tag in the (00000) configuration passes the sensor. Dashed line: Simulated AC bridge response. 